metropolitan US provide 1 bed per 1800 patient visits per year on average.

Objective: We sought to improve patient flow in our 24-bed ED at Santa Clara Valley Medical Center, a large tertiary public hospital in San Jose, California.

Methods: We applied a bundle of interventions based on Lean principles aimed at improving overcrowding in our department step-wise from October 2006 to December 2010, ranging from rapid medical evaluation to physician on arrival. We measured patient volume, left without being seen (LWBS) rates, time to provider (minutes), and time to discharge (minutes) as monthly averages. We secondarily measured CMS core measure performance for pneumonia and STEMI care and patient satisfaction scores.

Results: Our emergency patient volume doubled from an average of 192 to 412 per day (peak). Our LWBS rate decreased from 16.0 to $1.4 \%$. Our time to provider decreased from 86 to 32 minutes. Our time to discharge decreased from 210 to 145 minutes. Core measure performance improved to $100 \%$ for all five ED-related metrics. The percentage of patients who ranked their care as excellent increased from $30 \%$ to $45 \%$.

Conclusion: The bundle of interventions based on Lean principles applied in our ED appears to have enabled us to significantly improve multiple operational outcomes, despite a doubling of our patient volume in our low capacity ED. Posting a physician on arrival, as the first healthcare worker that a patient meets when they enter the ED, may have had the greatest impact on operational performance.

Prehosp Disaster Med 2011;26(Suppl. 1):s22-s23

doi:10.1017/S1049023X11000860

\section{(A82) Triage in the Prehospital Setting}

\section{A. Khorram-Manesh, ${ }^{1}$ A. Hedelin, ${ }^{2}$ P. Ortenwall ${ }^{2}$}

1. Research and Education, Gothenburg, Sweden

2. Gothenburg, Sweden

Introduction: The prehospital management of a patient starts with a telephone call to and triage by the ambulance dispatcher centre followed by continuous evaluations by ambulance crews and staff at emergency departments.

Aim: The aim of this study was to find out if these units have the same triage systems and if the initial evaluation matches the outcome at the hospital emergency departments.

Method and Material: Over 27000 ambulance transports within Gothenburg were studied by evaluating the ambulance medical records with regards to initial triage performed by the ambulance dispatcher centre using a medical index and triage performed by ambulance crews and staff at the emergency departments. Results: There was no common triage system between these units. We also found a discrepancy between the initial triage using the medical index and physiological-anatomical triage performed by ambulance crews and staff at the emergency departments. As an example 50\% of all patients triaged as priority one by the ambulance dispatcher centre were down-graded to priority $2-4$ by the other units involved.

Discussion and Conclusions: A mutual and standardized system for triage is needed. Although over-triaged by ambulance dispatcher centre may be medically motivated, the difference between priorities should be minimized to a medically accepted level (25-35\%).

Prehosp Disaster Med 2011;26(Suppl. 1):s23

doi:10.1017/S1049023X11000872

\section{(A83) Pediatric Disasters: Key Elements for Improving} Care

\section{S.E. Mace, ${ }^{1}$ C.T.J. Doyle ${ }^{2}$}

1. Emergency Services Institute, Cleveland, Ohio, United States of America

2. Emergency Department, Ann Arbor, Michigan, United States of America

$80 \%$ of children are seen in non-Pediatric Emergency Departments (EDs). In a disaster, most children and their caregivers will go to the closest or their regularly identified ED for treatment. In disasters, the preservation of the Pediatric Tertiary Infrastructure for the sickest and most injured children is critical. Surge capacity for pediatrics may involve both ante-grade and retrograde distribution of pediatric patients and health care staff to preserve Tertiary capacity. Reverse Triage of stable pediatric patients to other hospitals with adapted units and staff can decompress tertiary facilities. General hospitals can allow an expanded care for pediatric patients. Surge capacity needs to be addressed to allow non-pediatric facilities to surge for pediatric patients. Disaster Credentialing by immediate cross-credentialing of appropriate health care staff needs to be reciprocal and internet based to allow appropriate staff to attend pediatric patients. Pediatric consultants can augment healthcare staff to allow input into expanded care roles. Pre-hospital providers should have more pediatric training. Rotated regional caches of pediatric equipment would expedite safe pediatric disaster site care and pre-hospital transportation to definitive care. Pediatric patients should routinely be included in disaster drills and in all-inclusive disaster plans, rather than in separate drills and plans. Pediatric patients are usually accompanied by caregivers who may need care as well. Secure tracking and reunification of unaccompanied minors needs to be addressed to allow tracking across jurisdictional boundaries. Limited access to data on children, and credentialing of shelter staff would preclude access by anyone without a specific need to know. There are no clear uniform liability statutes for care in declared disasters as well as no uniform agreements for reimbursement for medical care. These issues are an important facet of disaster care that still needs to be addressed.

Prehosp Disaster Med 2011;26(Suppl. 1):s23

doi:10.1017/S1049023X11001257

(A84) Video Documentation as a Supplemental Teaching Tool for Multi-Focused Emergency Management Exercises

C. Tedeschi, ${ }^{1}$ L.M. Hilmi ${ }^{2}$

1. Emergency Medicine, New York, United States of America

2. Bel Air, United States of America

Introduction: Emergency management education and largescale disaster drills help to increase local capacity for dealing with a multitude of hazards. Video recordings of disaster drills offer participants with little exposure to fundamental emergency management principles an introduction to important topics and scenarios. Such resources allow ongoing training 